\title{
Clinical implications of intermittent Ramadan fasting on stable plaque psoriasis: a prospective observational study
}

\author{
Nawaf Almutairi', Dalia Shaaban ${ }^{2}$ \\ ${ }^{1}$ Department of Medicine, Faculty of Medicine, Kuwait University, Kuwait \\ ${ }^{2}$ Department of Dermatology, Farwaniya Hospital, Farwaniya, Kuwait
}

Adv Dermatol Allergol 2022; XXXIX (2): 368-374

\begin{abstract}
Introduction: Psoriasis is a chronic immune-mediated inflammatory disease. Environmental factors including diet have been supposed to play a role in its pathogenesis. Fasting in Ramadan consists of intermittent fasting in which participating Muslims refrain from eating and drinking from dawn up to sunset.

Aim: To validate the possible clinical consequences of Ramadan fasting for patients with psoriasis.

Material and methods: The study was conducted in Ramadan 2019. It included patients over 18 years who were diagnosed with stable chronic plaque psoriasis. PASI and BSA scores, body mass index and biochemical tests (including blood lipids, fasting blood glucose) were compared before and after a month of Ramadan fasting.

Results: The study included 121 psoriasis patients. The mean PASI score was $4.36 \pm 3.22$ at the beginning of the study, which was reduced to $3.51 \pm 1.26$ at the end of the study. The difference was statistically significant $(p=0.001)$. There was no difference in the change of weight. We found a statistically significant difference in fasting plasma glucose, HDL and triglycerides. Only mild adverse events were noted during the study period.

Conclusions: Ramadan intermittent fasting has beneficial effects on severity of the disease in psoriasis patients with a reduction in PASI score and no serious health hazards. Thus, Ramadan intermittent fasting could be considered during treatment of psoriasis patients.
\end{abstract}

Key words: Intermittent fasting, Ramadan, psoriasis.

\section{Introduction}

Psoriasis is a chronic immune-mediated inflammatory disease with a prevalence of about $2-3 \%$ all over the world [1, 2]. It is characterized by an accelerated tumour necrosis factor- $\alpha$ (TNF- $\alpha$ )/interleukin-23 (IL-23)/IL-17 axis, and hyperproliferation and abnormal differentiation of epidermal keratinocytes [3]. A group of genetic and environmental factors are elaborated in the pathogenesis of psoriasis and its comorbidities [4, 5].

Diet has been proposed to play a role in both the aetiology and pathogenesis of psoriasis [6]. Fasting periods, low-energy diets and vegetarian diets were found to improve psoriasis symptoms. Diets rich in omega-3 polyunsaturated fatty acids from fish oil also showed beneficial effects on psoriasis symptoms. These diets were suggested to modify the polyunsaturated fatty acid metabolism with suppression of the inflammatory processes involved in pathogenesis of psoriasis. Moreover, some patients with psoriasis might have an elevated sensitivity to gluten. Fasting periods, low-energy diets and vegetarian diets improved psoriasis symptoms in some studies [7]. Nutrition plays a principal role, by itself, either in the pathogenesis of psoriasis or in affecting drug pharmacokinetics and pharmacodynamics [6, 8, 9].

Intermittent fasting (IF) is an interventional approach where people are subjected to variable periods of fasting. IF has lately attracted consideration because experimental studies have highlighted its potential for regulating metabolic abnormalities [10]. This regimen has also revealed better adherence than other methods [11].

The month of Ramadan is the ninth month of the Muslim lunar calendar (al-Hijra), which has great significance and value among Muslims as it represents the month of the descent of the Noble Book (al-Qur'an). In Ramadan, Muslims must refrain from eating or drinking during daylight hours. During the Ramadan fast, Muslims eat two main meals, one before sunrise referred to as suhoor and the other shortly after sunset called iftar [12]. Islam encourages that the pre-dawn meal be as late

Address for correspondence: Prof. Dr. Nawaf Almutairi MD, MRCPC, Department of Medicine, Faculty of Medicine, Kuwait University, P.O. Box 280, Farwaniya 80000, Kuwait, e-mail: nalmut@usa.net Received: 20.03.2021, accepted: 29.03.2021. 
as possible so Muslims can better tolerate the fasting. People with sickness, ageing people, and pregnant and nursing women are excused [12].

The Ramadan fasting is not limited to abstinence from food and drinking, but also from smoking, and sexual intercourse. Inversely from other forms of fasting and diet regimens, the Ramadan fasting follows the circadian rhythm. The Ramadan fasting is a rather popular model of intermittent circadian fasting that is practiced every year by the Muslim population worldwide [12].

Some clinical implications of Ramadan fasting are well noted. Ramadan fasting has positive immunomodulatory effects in some immunological diseases such as rheumatic disorders [13] and psoriatic arthritis [14].

\section{Aim}

Data on the effects of Ramadan fasting on psoriasis, however, are not adequately acknowledged, so we aimed to carry out this study to validate the possible clinical consequences of Ramadan fasting for patients with psoriasis.

\section{Material and methods}

This study was an observational study conducted during Ramadan 2019, in the outpatient clinics of the Department of Dermatology at Farwaniya Hospital in Kuwait.

\section{Patients}

Patients were recruited from the 4 outpatient dermatology units at Farwaniya Hospital, Kuwait. The study included patients over 18 years who were diagnosed with chronic stable plaque psoriasis (patients with no new lesions and old lesions of the same size with Psoriasis Area and Severity Index "PASI" between two consecutive follow-ups < 10\%) and keen to fast during the month of Ramadan. All current medications were in the maintenance phase and not induction phase. Patients were excluded if they had a diagnosis of psoriasis other than chronic plaque (guttate, erythrodermic, pustular psoriasis and psoriatic arthritis), pregnant or lactating females and patients with any medical condition that could interfere with Ramadan fasting (e.g. evident diabetes and severe heart, liver or renal diseases).

\section{Methods}

Patient characteristics and data were documented in a standard form for each of the enrolled patient. The data included age, sex, age at onset, severity and duration of disease, details of past treatment, presence of comorbid conditions, family history of psoriasis, diabetes and hyperlipidaemia. For each of the enrolled patients, careful general medical examination was done and weight, height, body mass index, waist circumference, blood pressure were measured. Body mass index (BMI) was calculated as weight in $\mathrm{kg}$ divided by height in $\mathrm{m}^{2}$.

Laboratory tests including fasting blood glucose and serum lipid levels were done in each patient. Standard laboratory tests (complete blood count, liver and renal function tests, cholesterol, triglycerides (TG), uric acid, folic acid, homocysteine, and C-reactive protein) were also done. All biochemical markers were measured at the central laboratory of Farwaniya Hospital.

The severity of psoriasis was assessed, according to the body surface area (BSA) and PASI indexes, by the same dermatologist every week for 5 weeks. The PASI score accounts for the extent of psoriatic involvement of the body surface area on the head, trunk, arms and legs, as well as the severity of scale formation, erythema and plaque induration on each region of the body.

All clinical and laboratory measurements were performed at the baseline visit (3 days before starting fasting in Ramadan) and repeated 3 days after the end of Ramadan (after Eid Al Fitr Holiday) and any reduction was reported.

Adverse events, such as dry mouth, diarrhoea, constipation, fatigue, increased cold sensitivity, headache, hunger, and visual disturbance were reported at each visit.

\section{Statistical analysis}

Continuous variables were presented as medians with inter-quartile ranges (IQR), while categorical variables were presented as numbers with percentages. The 95\% confidence interval $(\mathrm{Cl})$ was calculated for each outcome measure. For descriptive purposes, continuous variables were also categorized using clinically relevant thresholds. Differences between arms were tested using the Mann-Whitney $U$-test for continuous variables and Pearson's $\chi^{2}$-test for categorical variables. Associations between continuous variables were assessed using Spearman's rank correlation coefficient $(\rho)$. Two-sided $p$-values of $<0.005$ were considered to indicate statistical significance in all tests.

\section{Results}

The study included 121 psoriasis patients (75 males, 46 females). It was carried out during Ramadan 2019 which started on Monday, 6 May 2019 and ended on Tuesday, 4 June 2019, with a length of fasting of approximately 14 h. Patients' ages ranged from 19 to 61 with a mean of $43.5 \pm 4.2$ years, with a mean duration of psoriasis disease of $9.3 \pm 7.8$ years.

Topical medications including emollients, steroids, keratolytic agents and vitamin D derivatives were used by 39 (32.23\%) patients. Regarding the systemic treatment, $15(12.93 \%)$ patients were treated with methotrexate, 25 (20.66\%) patients were treated with adalimumab 40 mg, 19 (15.70\%) patients were treated with ixekizumab $80 \mathrm{mg}, 14$ (11.57\%) patients were treated with etan- 
Table 1. Characteristics of patients at baseline

\begin{tabular}{|c|c|}
\hline Characteristics & Value \\
\hline \multicolumn{2}{|l|}{ Sex, $n(\%):$} \\
\hline Male & $75(61.98)$ \\
\hline Female & $46(38.02)$ \\
\hline Age at enrolment [years] mean \pm SD & $43.5 \pm 4.2$ \\
\hline Duration of psoriasis [years] mean \pm SD & $9.3 \pm 7.8$ \\
\hline Psoriasis severity (PASI score) mean \pm SD & $4.36 \pm 3.22$ \\
\hline Family history of psoriasis, $n(\%)$ & 17 (14.05) \\
\hline $\begin{array}{l}\text { Waist circumference greater than } 90^{\text {th }} \text { percentile, } \\
n(\%)\end{array}$ & $81(66.94)$ \\
\hline $\mathrm{BMI}\left[\mathrm{kg} / \mathrm{m}^{2}\right]$ & $37.8 \pm 2.4$ \\
\hline BSA (range, median) & $2 \%: 12 \%, 3.45$ \\
\hline Triglyceridemia, $n$ (\%) & $63(52.07)$ \\
\hline Low HDL cholesterol $\geq 150$ mg/dl, $n$ (\%) & $31(25.62)$ \\
\hline Fasting blood glucose $\geq 100 \mathrm{mg} / \mathrm{dl}, n(\%)$ & $47(38.84)$ \\
\hline High blood pressure, $n(\%)$ & $15(12.40)$ \\
\hline \multicolumn{2}{|l|}{ Treatment, $n$ (\%): } \\
\hline Topical & $39(32.23)$ \\
\hline Methotrexate & $15(12.39)$ \\
\hline \multicolumn{2}{|l|}{ TNF blockers: } \\
\hline Adalimumab 40 mg/week & $25(20.66)$ \\
\hline Etanercept 50 mg/week & $14(11.57)$ \\
\hline \multicolumn{2}{|l|}{ IL-17 blockers: } \\
\hline Ixekizumab 80 mg/4 weeks & $19(15.70)$ \\
\hline Secukinumab 150 mg/4 weeks & $26(21.49)$ \\
\hline
\end{tabular}

PASI - Psoriasis Area and Severity Index, BMI - body mass index, HDL - highdensity lipoprotein.

PASI, mean \pm SD

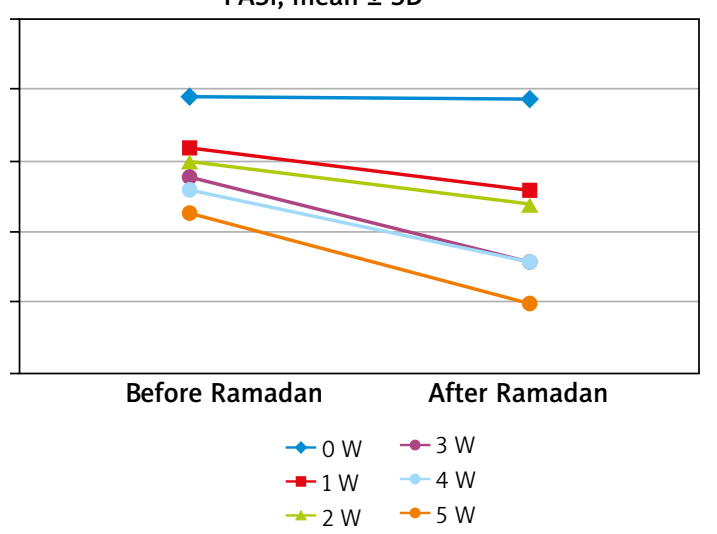

Figure 1. Mean $( \pm S D)$ Psoriasis Area and Severity Index (PASI) scores from baseline (before Ramadan fasting) to week 5 (after Ramadan fasting)

ercept $50 \mathrm{mg}$, and 9 (7.43\%) patients were treated with secukinumab $300 \mathrm{mg}$.
The average BMI of the included patients was 37.8 $\pm 2.4 \mathrm{~kg} / \mathrm{m}^{2}$. Forty-nine (40.50\%) patients were normal in weight, while 60 (49.59\%) patients were overweight and 12 (9.91\%) patients were obese. Waist circumference greater than $90^{\text {th }}$ percentile was noted in 81 (66.94\%) patients.

PASI scores of the patients before fasting ranged from 1.82 to 10.73 with a mean of $4.36 \pm 3.22$. BSA ranged from $2 \%$ to $12 \%$, with a median of $3.45 \%$. TG levels $\geq 150 \mathrm{mg} /$ dl were found in 63 (52.07\%) patients, while levels of fasting glucose $\geq 100 \mathrm{mg} / \mathrm{dl}$ were noticed in 47 (38.84\%) patients (Table 1).

\section{After completing the study}

There was no statistically significant difference in the change of weight noticed after Ramadan fasting in 102 (84.30\%) patients, whereas 14 (11.57\%) subjects gained $1 \mathrm{~kg}$ and 5 (4.13\%) individuals gained $2 \mathrm{~kg}$. However, no patient recorded loss of weight. There were also no statistically significant differences regarding raised blood pressure before and after the end of the study.

PASI was reduced to $3.51 \pm 1.26$ with a mean difference of $0.85 \pm 1.32$ and the difference was statistically significant $(p=0.001)$. The mean values of PASI score were reduced with time during the 5 weeks' study period as shown in Figure 1. The improvements in PASI occurred regardless of age, disease duration, clinical symptoms, and family history for psoriasis.

The reduction in PASI score after the Ramadan fasting was also not significantly related to the type of treatment received by the patients although patients received ixekizumab reported more reduction $(p>0.05)$.

The Ramadan fasting had no impact on any biochemical parameters studied except for fasting plasma glucose, HDL and triglycerides. We found a statistically significant correlation with fasting plasma glucose, HDL and triglycerides. Raised triglycerides levels were significantly more prevalent before fasting, with 63 (52.07\%) patients having TG levels $\geq 150 \mathrm{mg} / \mathrm{dl}$, when compared to 51 (42.15\%) after the fasting period. Similarly, a statistically significant difference in the levels of fasting glucose was noticed, with 47 (38.84\%) patients before fasting having $\geq 100 \mathrm{mg} / \mathrm{dl}$ against 31 (25.62\%) patients after fasting (Table 2).

Only mild adverse events were noted during the study period. Twenty-one patients complained of feelings of hunger, especially in the early days of fasting and 7 patients sometimes felt fatigue during the fasting days. Constipation was reported by 11 patients.

\section{Discussion}

This is an observational study of the effects of Ramadan IF on psoriasis patients. Only patients with stable psoriasis were enrolled in this study, so that any clinical change in the disease could be attributed to nutritional 
Table 2. Serum biochemical parameters before and after Ramadan intermittent fasting

\begin{tabular}{|c|c|c|c|}
\hline Parameter & Before fasting & After fasting & $P$-value \\
\hline $\mathrm{WBC}\left[\times 10^{3} / \mathrm{ml}\right]$ mean $\pm \mathrm{SD}$ & $8.37 \pm 1.8$ & $8.53 \pm 2.4$ & 0.30 \\
\hline $\mathrm{RBC}\left[\times 10^{6} / \mu \mathrm{l}\right]$ mean $\pm \mathrm{S} . \mathrm{D}$ & $5.4 \pm 1.7$ & $5.6 \pm 1.5$ & 0.26 \\
\hline $\mathrm{Hb}[\mathrm{g} / \mathrm{dl}]$ mean $\pm \mathrm{S} . \mathrm{D}$ & $14.58 \pm 1.5$ & $14.27 \pm 1.2$ & 0.23 \\
\hline Platelets $[1,000 / \mu \mathrm{l}]$ mean \pm S.D & $214 \pm 37$ & $217 \pm 49$ & 0.21 \\
\hline \multicolumn{4}{|l|}{ Kidney profile: } \\
\hline Creatinine $[\mathrm{mmol} / \mathrm{l}]$ mean $\pm \mathrm{SD}$ & $79.38 \pm 11.4$ & $81.42 \pm 12.7$ & 0.01 \\
\hline Urea $[\mathrm{mg} / \mathrm{dl}]$ mean $\pm \mathrm{SD}$ & $7.21 \pm 1.8$ & $6.75 \pm 1.6$ & 0.27 \\
\hline \multicolumn{4}{|l|}{ Liver profile: } \\
\hline AST $[U / I]$ mean \pm SD & $22.37 \pm 7.1$ & $21.81 \pm 6.9$ & 0.054 \\
\hline ALT $[U / I]$ mean $\pm S D$ & $29.41 \pm 9.8$ & $28.71 \pm 12.9$ & 0.65 \\
\hline Uric acid $[\mathrm{mg} / \mathrm{dll}]$ mean $\pm \mathrm{SD}$ & $6.34 \pm 4.1$ & $7.25 \pm 3.4$ & 0.03 \\
\hline \multicolumn{4}{|l|}{ Lipid profile: } \\
\hline Triglycerides $[\mathrm{mg} / \mathrm{dl}]$ mean \pm SD & $158.37 \pm 84.2$ & $124.8 \pm 55.1$ & 0.019 \\
\hline Triglyceridemia $n(\%)$ & $63(52.07)$ & $51(42.15)$ & 0.005 \\
\hline Total cholesterol [mg/dl] mean \pm SD & $218.0 \pm 67.3$ & $213.0 \pm 57.0$ & 0.748 \\
\hline LDL cholesterol $[\mathrm{mg} / \mathrm{dl}]$ mean \pm SD & $137.4 \pm 37.6$ & $131.2 \pm 48.3$ & 0.219 \\
\hline HDL cholesterol [mg/dl] mean \pm SD & $42.3 \pm 12.2$ & $56.3 \pm 17.5$ & 0.006 \\
\hline Fasting blood sugar $[\mathrm{mg} / \mathrm{dll}]$ mean $\pm \mathrm{SD}$ & $128.17 \pm 08$ & $112.57 \pm 36.13$ & 0.007 \\
\hline Raised fasting blood sugar $n(\%)$ & $47(38.84)$ & $31(25.62)$ & 0.005 \\
\hline
\end{tabular}

$W B C$ - white blood cells, RBC - red blood cells, Hb-haemoglobin, AST - aspartate aminotransferase, ALT - alanine aminotransferase, LDL - low-density lipoprotein, $H D L$ - high-density lipoprotein.

changes during the month of Ramadan fasting rather than to the effect of treatment received.

The median PASI score at the end of fasting (week 5) in our study cohort was significantly reduced from 4.36 \pm 3.22 before Ramadan fasting to $3.51 \pm 1.26$ at the end of the study. This was in agreement with Damiani et al. [15], who presented a recent study investigating the advantageous effects of Ramadan fasting on the activity of psoriasis diseases expressed in PASI. A significant decrease in the PASI score after the Ramadan fasting was found. Adawi et al. [14] also reported improvement in psoriatic arthritis disease activity after Ramadan fasting

Dietary restriction in the form of intermittent bouts of fasting with without chronic caloric restriction has been revealed to exert a host of health benefits associated with chronic inflammatory states [16, 17]. Intermittent fasting has been shown to improve many of immunological disturbances in psoriasis, even inflammatory parameters such as TNF and CRP [7]. The significant reduction in PASI after diet/fasting seems to reflect the inhibition of cellular proliferation and of release of cell activation products, including pro-inflammatory mediators [18].

Adawi et al. [12] systematically reviewed 45 studies that assessed the effect of fasting on immunity during the month of Ramadan. They concluded that Ramadan IF could have an immunomodulatory effect and can decrease the levels of several proinflammatory cytokines levels such as IL-1, TNF, and IL-6 which return to the basal pre-Ramadan status shortly afterwards [12].

Aksungar et al. [19] measured inflammatory markers such as interleukin-6, CRP and biochemical parameters during prolonged IF in Ramadan. The results demonstrated a decrease in the inflammatory response since plasma levels of IL-6 and CRP were consistently reduced by fasting. Similarly, Faris et al. [20] found a significant decrease in serum levels of the proinflammatory cytokines, IL-6, IL-1 $\beta$, TNF- $\alpha$, after Ramadan fasting. Akrami et al. [21] showed that pro-inflammatory chemokines (CXCL1, CXCL10) and the constituent chemokine (CXCL12) were downregulated during fasting. These chemokines are identified to be downstream targets of TNF- $\alpha$. Thus, fasting has been shown to play a role in controlling inflammation via chemokines.

Short-term fasting may yield these benefits by negatively regulating systemic and/or white adipose tissue-related proinflammatory cytokine expression, and because of its intense consequence on immunomodulatory hormones such as insulin, leptin, and corticosterone [22, 23].

Tridon et al. [24] also reported a noteworthy decrease in the percentage of T-cells subsets in his study populations after 36-hour fasting period and this reduction continued after the refeeding. This may elucidate some of the advantageous effects induced by fasting in psoriasis 


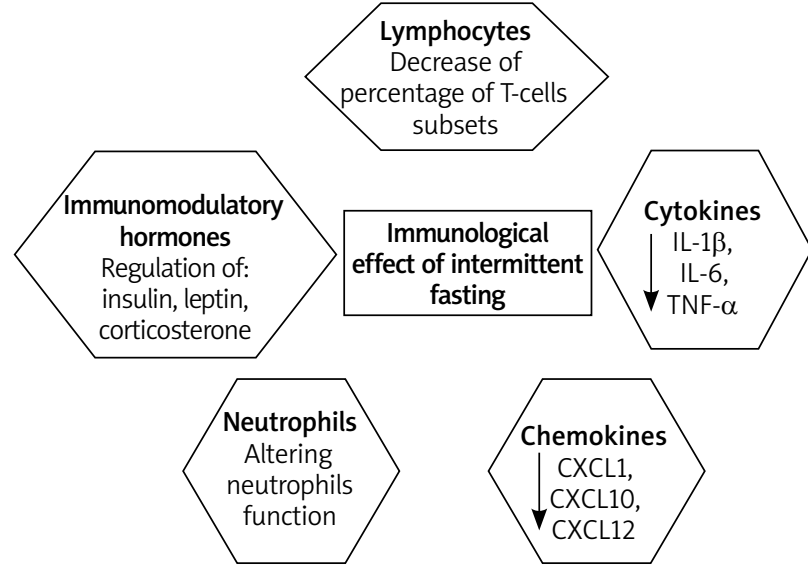

Figure 2. Effect of fasting on the individual elements of inflammation in psoriasis

patients as T-cells are the chief performer in the disease (Figure 2).

The immunological effect of fasting was also studied in some immunologic diseases other than psoriasis. Hafstrom et al. [25] investigated the effects of fasting on disease activity, neutrophil function, fatty acid composition, and leukotriene biosynthesis in patients with rheumatoid arthritis. They found that fasting can influence neutrophils function by attenuating the release of myeloperoxidase enzyme, decrease of the production of leukotrienes, and altering the fatty acid composition of membrane phospholipids leading to the reduction of inflammatory markers and symptoms [25]. Lithell et al. [26] reported an improvement of two chronic inflammatory dermatoses, atopic dermatitis and pustulosis palmaris et plantaris, with IF for 2 weeks [26].

Both central and peripheral clocks act to preserve the circadian rhythm of different tissue physiologically via controlling "tissue-specific gene expression" [27]. In 2018 Couto et al. reported that magnitude of adipose and skin response to time of the day was around twice that of fasting hours [28].

Damiani et al. [15] explained that the reduction of PASI score after Ramadan fasting reflects the influence of dieting strategy, the biological clock, and circadian rhythm of Ramadan IF on the treatment of plaque psoriasis. Most Muslims make a shift in their sleep cycle, so they sleep during the fasting time of the day and work and eat during the night. Moreover, patients tend to administer their drugs during the night and this may have beneficial effects as several studies have suggested that bedtime or night doses compared with morning administration can lead to a better effect of the drug. Thus, the impact of the Ramadan fasting on psoriasis could therefore be largely due to the change in sleep architecture and dynamics/patterns, rather than to the times of the fasting and re-feeding [15].
We found no decrease in body weight after fasting. There was no difference in the change of weight noticed after Ramadan fasting in $84.30 \%$ of the patients, while 14 patients only showed increase in weight. Shehab et al. [29], investigating the effects of Ramadan fasting on body weight, found that there were losses both in body weight and waist circumference immediately following Ramadan, with a significant reduction of BMI from $28.1 \mathrm{~kg} / \mathrm{m}^{2}$ before fasting to $27.2 \mathrm{~kg} / \mathrm{m}^{2}$ after fasting. However, Kamble and Hiremath [30] observed a mild non-significant reduction of body weight and BMI after Ramadan fasting. Improving PASI score regardless of the change in the patients' weight may support that the fasting process itself can affect the immune system activity with improvement in disease activity [12].

Serum biochemical parameters after Ramadan IF in our study population showed that serum levels of TG, HDL cholesterol, and fasting blood sugar were significantly improved as compared to baseline. However, there were no significant differences from baseline in total cholesterol and LDL cholesterol.

Kamble and Hiremath [30] inspected the consequence of fasting on 30 healthy volunteers. They found that fasting significantly decreased low-density lipoprotein. Furthermore, fasting considerably increased high-density lipoprotein, while there was no noteworthy change in glucose, insulin, and insulin resistance levels.

Aksungar et al. [31] assessed cardiovascular health in Muslim individuals during Ramadan, with special emphasis on coagulation. The results showed improvements in the lipid profile, with increased HDL-cholesterol levels and decreased values of HDL risk factor (CT/HDL), during the fast and 20 days after it.

Kerndt et al. [32] inspected the metabolic effects of long-term fasting in human subjects who experienced a 36-day complete fasting schedule for religious reasons. Plasma glucose levels dropped immediately at the beginning of fasting and remained low throughout the fasting period. Shehab et al. [29] found that there was a significant increase in HDL and a decrease in LDL cholesterol levels a month after Ramadan fasting.

Numerous studies displayed a decrease in TG levels during Ramadan fasting [29, 31, 33-36]. Afandi et al. [37] reported that fasting for a month resulted in a significant decrease in blood glucose levels, while high-density lipoprotein levels significantly increased in their study group at the end of the fasting period. These results suggest that Ramadan fasting may be a suitable lifestyle modification to improve a blood lipid profile $[29,38]$.

There were no serious risks for health recorded in our psoriasis patients due to fasting. Twenty-one patients complained of feelings of hunger and 7 patients sometimes felt fatigue during the fasting days. Constipation was reported by 11 patients. Similarly, Damiani et al. [18] did not record any serious risks in their study cohort during IF of Ramadan. 
To the best of our knowledge, this is the first study in the Arab area that investigated the effect of IF Ramadan fasting on patients with psoriasis.

The limitation of this is that we could not control the type and amount of food consumed by the patients. In addition, 1 month of fasting is a relatively short duration to report all the changes induced by fasting. Further studies on larger groups of patients and longer duration of fasting are needed to confirm the results.

\section{Conclusions}

This study demonstrated that Ramadan IF has beneficial effects on severity of the disease in psoriasis patients with a reduction in PASI score. No serious health hazards were reported in psoriasis patients as a result of Ramadan IF. Thus, Ramadan IF could be considered during treatment of psoriasis patients.

\section{Conflict of interest}

The authors declare no conflict of interest.

\section{References}

1. Gelfand JM, Weinstein R, Porter SB, et al. Prevalence and treatment of psoriasis in the United Kingdom: a populationbased study. Arch Dermatol 2005; 141: 1537-41.

2. Barker J. Skin diseases with high public health impact. Psoriasis. Eur J Dermatol 2007; 17: 563-4.

3. Al-Mutairi N, Al-Farag S, Al-Mutairi A, et al. Comorbidities associated with psoriasis: an experience from the Middle East. J Dermatol 2010; 37: 146-55.

4. Zeng J, Luo S, Huang Y, et al. Critical role of environmental factors in the pathogenesis of psoriasis. J Dermatol 2017; 44: 863-72.

5. Singh S, Pradhan D, Puri P, et al. Genomic alterations driving psoriasis pathogenesis. Gene 2019; 683: 61-71.

6. Wolters $M$. The significance of diet and associated factors in psoriasis. Hautarzt 2006; 57: 999-1004.

7. Wolters M. Diet and psoriasis: experimental data and clinical evidence. Br J Dermatol 2005; 153: 706-14.

8. Carrascosa JM, Rocamora V, Fernandez-Torres RM, et al. Obesity and psoriasis: Inflammatory nature of obesity, relationship between psoriasis and obesity, and therapeutic implications. Actas Dermosifiliogr 2014; 105: 31-44.

9. Upala S, Sanguankeo A. Effect of lifestyle weight loss intervention on disease severity in patients with psoriasis: a systematic review and meta-analysis. Int J Obes 2015; 39: 1197-202.

10. Anson RM, Guo Z, Cabo R, et al. Intermittent fasting dissociates beneficial effects of dietary restriction on glucose metabolism and neuronal resistance to injury from calorie intake. Proc Natl Acad Sci USA 2003; 100: 6216-20.

11. Varady KA, Bhutani S, Church EC, et al. Short-term modified alternate-day fasting: a novel dietary strategy for weight loss and cardioprotection in obese adults. Am J Clin Nutr 2009; 90: 1138-43.

12. Adawi M, Watad A, Brown S, et al. Ramadan fasting exerts immuno-modulatory effects: insights from a systematic review. Front Immunol 2017; 8: 1144.
13. Bragazzi NL, Watad A. The impact of fasting on rheumatic diseases. Isr Med Assoc J 2017; 19: 378-9.

14. Adawi M, Damiani G, Bragazzi NL, et al. The impact of intermittent fasting (Ramadan fasting) on psoriatic arthritis disease activity, Lee enthesitis, and dactylitis: a multicentre study. Nutrients 2019; 11: 601.

15. Damiani G, Watad A, Bridgewood C, et al. The impact of Ramadan fasting on the reduction of PASI score, in moderateto-severe psoriatic patients: a real-life multicenter study. Nutrients 2019; 11: 277.

16. Longo VD, Mattson MP. Fasting: molecular mechanisms and clinical applications. Cell Metabolism 2014; 19: 181-92.

17. Lee C, Longo V. Dietary restriction with and without caloric restriction for healthy aging. Version1.F1000Res 2016; 5: F1000.

18. Coimbra S, Oliveira H, Reis F, et al. Circulating adipokine levels in Portuguese patients with psoriasis vulgaris according to body mass index, severity and therapy. J Eur Acad Dermatol Venereol 2010; 24: 1386-94.

19. Aksungar FB, Topkaya AE, Akyildiz M. Interleukin-6, C-reactive protein and biochemical parameters during prolonged intermittent fasting. Ann Nutr Metab 2007; 51: 88-95.

20. Faris MA, Kacimi S, Al-Kurd RA, et al. Intermittent fasting during Ramadan attenuates proinflammatory cytokines and immune cells in healthy subjects. Nutr Res 2012; 32: 947-55.

21. Akrami MF, Ahmadi Z, Hassanshahi G, et al. Dose Ramadan fasting affects inflammatory responses: evidences for modulatory roles of this unique nutritional status via chemokine network. Iran J Basic Med Sci 2013; 16: 1217-22.

22. Hershock D, Vogel WH. The effects of immobilization stress on serum triglycerides, nonesterified fatty acids, and total cholesterol in male rats after dietary modifications. Life Sciences 1989; 45: 157-65.

23. Otero M, Lago R, Gomez R, et al. Towards a pro-inflammatory and immunomodulatory emerging role of leptin. Rheumatology 2006; 45: 944-50.

24. Tridon A, Beaufrère B, Caldefie F, et al. Specific and nonspecific immune responses to fasting and refeeding differ in healthy young adult and elderly persons. Am I Clin Nutr 2001; 74: 670-8.

25. Hafstrom I, Ringertz B, Gyllenhammar H, et al. Effects of fasting on disease activity, neutrophil function, fatty acid composition, and leukotriene biosynthesis in patients with rheumatoid arthritis. Arthritis Rheum 1988; 31: 585-92.

26. Lithell H, Bruce A, Gustafsson IB, et al. A fasting and vegetarian diet treatment trial on chronic inflammatory disorders. Acta Derm Venereol 1983; 63: 397-403.

27. Van Laake LW, Lüscher TF, Young ME. The circadian clock in cardiovascular regulation and disease: lessons from the Nobel Prize in physiology or medicine 2017. Eur Heart J 2018; 39: 2326-9.

28. Couto AA, Glastonbury CA, El-Sayed JS, Small KS. Fasting and time of day independently modulate circadian rhythm relevant gene expression in adipose and skin tissue. BMC Genomics 2018; 19: 659.

29. Shehab A, Abdulle A, El Issa A, et al. Favorable changes in lipid profile: the effects of fasting after Ramadan. PLoS One 2012; 7: e47615.

30. Kamble S, Hiremath S. Insulin resistance and blood lipid levels during fasting. Natl J Physiol Pharm Pharmacol 2018; 8: 1158-61.

31. Aksungar FB, Eren A, Ure S, et al. Effects of intermittent fasting on serum lipid levels, coagulation status and plasma homocysteine levels. Ann Nutr Metab 2005; 49: 77-82. 
32. Kerndt PR, Naughton JL, Driscoll CE, et al. Fasting: the history, pathophysiology and complications. West I Med 1982; 137: 379-99.

33. Mahoob S, Sattarivand R, Nouri M, et al. Effect of Ramadan fasting on serum lipid profiles in normal and hyperlipidemic subjects. Saudi Med J 1999; 20: 947-50.

34. Asgary S, Aghaei F, Naderi GA, et al. Effects of Ramadan fasting on lipid peroxidation, serum lipoproteins and fasting blood sugar. Med J Islamic World Acad Sci 2000; 13: 35-8.

35. Furuncuoglu Y, Karaca E, Aras S, et al. Metabolic, biochemical and psychiatric alterations in healthy subjects during Ramadan. Pak J Nutr 2007; 6: 209-11.

36. Haouari-Oukerro F, Ben-Attia M, Kaâbachi N, et al. Ramadan fasting influences on food intake consumption, sleep schedule, body weight and some plasma parameters in healthy fasting volunteers. Afr J Biotechnol 2013; 12: 3327-32.

37. Afandi BO, Hassanein MM, Majd LM, et al. Impact of Ramadan fasting on glucose levels in women with gestational diabetes mellitus treated with diet alone or diet plus metformin: a continuous glucose monitoring study. BMJ Open Diabetes Res Care 2017; 5: e000470.

38. Akaberi A, Golshan A, Moojdekanloo M, et al. Does fasting in Ramadan ameliorate lipid profile? A prospective observational study. Pak J Med Sci 2014; 30: 708-11. 